https://doi.org/10.18778/2196-8403.2021.06

TORSTEN VOß

\title{
HermanN Lenz und der Eugen Rapp-Zyklus als Narrativierung des literarischen Feldes der Bundes- republik?
}

Mag Hegel angesichts der allumfassenden Ökonomisierung und Marktregulation des künstlerischen Feldes in seinen Vorlesungen über die Ästhetik bereits im frühen 19. Jahrhundert vom Tod der Kunst gesprochen haben, Künstler und Künstlerinnen sind bis heute zum Glück noch nicht gestorben bzw. verschwunden. Paratexte und Interviews zeigen Versuche sich auch unter poststrukturalistischen, postsubjektiven und postfaktischen Vorzeichen auktorial durchzusetzen und auf dem Markt, aber doch auch ganz klar für sich, Aufmerksamkeit über die Auto-Präsentation in der Öffentlichkeit als Anhäufung von symbolischem Kapital zu produzieren bzw. diese Prozesse als Narrative auszugestalten.

Dass sich dabei auch immer eine Reflexionsebene gegenüber dem literarischen Feld ergibt, ja letzteres und damit den Literaturbetrieb zum Narrativ werden lässt, wird anhand von HERMANN LENZ Literaturbetriebsromanen genauer herausgearbeitet. Durch die Auseinandersetzung mit dem Literaturbetrieb ist es LENZ möglich, die Inszenierung und Selbstbehauptung von Autorschaft und Autorschaftsbewusstsein zu reanimieren.

Schlüsselwörter: HERMANN LENZ, Literaturbetriebsroman, Autofiktion, Paratext, Narrativ, literarisches Feld

Hermann Lenz and the Eugene Rapp novel series - an attempt to narrate the literary field of the Federal Republic of Germany?

Faced with the omnipresent economization and market regulation of the artistic field, Hegel had already discussed the death of art in the early $19^{\text {th }}$ century in his Vorlesungen über die Ästhetik; yet the artist was hardly dead, and remains alive today. Paratexts and interviews show artists' attempts at self-creation - in many contexts, including poststructural, post-subjective and post-factual. The goal is to attract attention through selfpresentation in public and gathering symbolic capital - for the sake of the market, or the artist himself, alternatively to transform these processes into semi-fictional narratives. 
The fact that reflection is always present in the literary sphere is analyzed in HERMANN LENZ'S novels revolving around the literary business. By confronting literary life, LENZ was able to reanimate the sense of autonomy and awareness of authorship.

Keywords: HERmAnn LenZ, literary-business novels, autofiction, paratext, narrative, literary field

\section{Hermann Lenz i cykl powieściowy o Eugenie Rappie jako próba opowiedzenia pola literackiego Republiki Federalnej Niemiec}

Choć Hegel w swych wykładach z estetyki pisał już na początku XIX w. - w związku z powszechną merkantylizacją i urynkowieniem kultury - o śmierci sztuki, twórczynie i twórcy przetrwali na szczęście do dziś dnia. Parateksty i wywiady świadczą o próbach utwierdzenia swojej autorskiej pozycji. Nawet w uwarunkowaniach postrukturalistycznych, postsubjektywnych i postprawdy chodzi o przyciąganie uwagi poprzez autoprezentację w przestrzeni publicznej i gromadzenie kapitału symbolicznego, ewentualnie kształtowanie tych procesów w toku narracji - na potrzeby rynku (sztuki) lub po prostu jedynie dla siebie. W tym kontekście rodzi się również refleksja na temat pola literackiego oraz tworzy narracja o nim i o produkcji literackiej. Artykuł prezentuje ten fenomen na przykładzie powieści HeRmanna LenZA. Dzięki konfrontacji z życiem literackim udało się LENZOWI ożywić autonomiczność i świadomość autorskości.

Slowa klucze: Hermann LenZ, powieść o produkcji literackiej, autofikcja, paratekst, narracja, pole literackie

\section{Vorab: Bis Peter Handke kam? Der Schriftsteller HERMANN LENZ und seine feldexterne (De-)Positionierung}

HERMANN LENZ (1913-1998) ist heute so gut wie aus dem literarischen Feld verschwunden. Der lange Zeit ausgesprochen produktive Romancier ist kaum noch Gegenstand der germanistischen Forschung, geschweige denn privater Lektüren. Ein früher Band in der, edition text \& kritik' stellt eines der wenigen Zeugnisse von philologischer Auseinandersetzung mit dem umfangreichen Werk des Autors dar. ${ }^{1}$ Er zählt mittlerweile zu den großen Unbekannten des

1 In dem von Klaus Briegleb und Sigrid Weigel herausgegebenen Band zur Gegenwartsliteratur seit 1968 in Hansers Sozialgeschichte der deutschen Literatur findet HERMANN LENZ beispielsweise nur an vier Stellen eine knappe Erwähnung des Autors. Briegleb selbst bezeichnet vor dem Hintergrund des NS-Terrors und politischer Verantwortung von Intellektuellen und Schriftstellern LENZ' Prosa recht kritisch als „Idyllentexte zur Harmonisierung des Erzählens“ (BRIEGLEB 1992:380). Zur Wirkungsgeschichte vgl. den Band von KREUZER / KREUZER (1981). Als Sekundärliteratur sind vor allem Rezensionen zu nennen, wenn auch von namhaften Vertretern wie Jürgen Manthey, Paul Konrad Kurz oder Peter Härtling. 
Literaturbetriebs und bietet daher genug Trouvaillen. Sein Romanzyklus über sein alter Ego, den Schriftsteller Eugen Rapp, beginnend mit dem Roman Verlassene Zimmer (1966), stellt nicht nur eine Selbstbeschreibung des eigenen Verständnisses von Autorschaft dar, sondern gibt auch Einblick in die Verhältnisse bundesrepublikanischer Wirklichkeit bzw. der unmittelbaren Nachkriegszeit und lässt den dargestellten Autor mit den Verfahren der Autofiktion ${ }^{2}$ und des Schlüsselromans ${ }^{3}$ zum Beobachter, Chronisten und Erzähler der Zeitgeschichte werden. Auch wenn diese keine Schriftstellerfiguren in ihren Mittelpunkt gestellt hatten, sondern eher die eigene Familienbiographie, ähnelt der Eugen RappZyklus doch sehr stark anderen Verfahren des chronistischen Erzählens, wie man es von Horst Bienek, Walter Kempowski, ${ }^{4}$ Peter Kurzeck oder Gerhard Henschel kennt. Das Allgemeine der Universalgeschichte kondensiert sich in einem individuellen Erleben über mehrere Dekaden. ${ }^{5}$

Der Rapp-Zyklus von HeRMANN LENZ zählt neun Bände. Auch von Feuilleton und Literaturkritik erst spät gefeiert, zeichnete sich das Erzählwerk des Autors nicht gerade über eine breite Rezeption innerhalb der Leserschaft aus. Dies könnte mit der Sperrigkeit und der ausgedehnt-nuancierten Beschreibungskunst seiner Erzählverfahren zusammenhängen, die - ganz unzeitgemäß - geradezu zur Ent-

2 Vgl. zum Genre den Sammelband von Wagner-EgelHaAf (2013).

3 Als neueste Gesamtdarstellung und Gattungstheorie vgl. JOHANNES FranZEN (2018) sowie die Aufsatzsammlung von GERTRUd MARIA RÖSCH (2005).

Eine Ausnahme stellen freilich die beiden Romane um den semi-fiktiven Schriftsteller Alexander Sowtschik dar, also Hundstage (1988) und Letzte Grüße (2003). Auch diese inszenieren einen Autor jenseits des Mainstreams und eine Perspektivierung des Literaturbetriebs aufgrund seiner Position.

Ähnliches gilt für die parallel und in einem Zeitraum entstandene Trilogie Im inneren Bezirk (1961-1980), welches das Schicksal der Offiziersfamilie von Sy durch die Augen der Tochter Margot von den 1930er Jahren bis in die früher Bundesrepublik schildert und vor dem Wechsel der politischen Systeme ein komplexes VaterTochter-Verhältnis organisiert. Auch hier stehen die Komplexe ,Vergangenheit', ,Heimat', ,Familie' und ,Natur' immer wieder im Mittelpunkt der Narration. Die Lyrizität mancher Beschreibungen der Naturwahrnehmungen Margots geben die Unmittelbarkeit der Eindrücke wieder, mit denen der Autor LENZ auch in seinen Gedichten Unverfälschtheit anzustreben versucht. Diese Eindrücke und die Bindung an den Vater stellen für die Protagonistin dann auch den titelgebenden inneren Bezirk dar, in den es sich vor der allgemeinen Bedrohung durch den Nationalsozialismus und auch die Zeit zurückzuziehen gilt. Dadurch meint der Titel inneren Bezirk hier mehr als nur ,innere Emigration'. Es geht um eine existentielle, metaphysische und anthropologische Aufladung des Terminus. 
schleunigung, zum Anhalten ja zum Verweilen einzuladen scheinen bzw. ihre genaue Beobachtungsgabe von zwischenmenschlichen Beziehungen, gesellschaftlichen Verhältnissen, aber auch scheinbar einfachsten pointilistischen Naturimpressionen dem Leser aufzubürden versuchen, abseits aller Aufmerksamkeit erzeugenden Effekte und eines unmittelbaren Gegenwartsbezugs.

Mit HERMANN LENZ begibt man sich nicht auf den Fast Track. Wenn bei ihm ein Bezug zur Gegenwart gegeben ist, dann ist dieser allein aus dem subjektiven Erleben auf einer auktorialen Erzählebene heraus konstruiert, bis zu dessen Berührungen mit den konstituierenden Mechanismen des literarischen Feldes und all seiner Repräsentanten und führenden Steuermänner. So muss man beim Lesen ebenfalls anhalten und rasches Umblättern vermeiden, um sich dieser Ästhetik der Langsamkeit hingeben zu können. ${ }^{6}$ Man könnte an dieser Stelle vielleicht literaturkritisch zugespitzt anmerken: Um sich an HERMANN LENZ erfreuen zu können, sollte man sich vorher vielleicht mit Raabe oder Stifter auseinandergesetzt haben. Es verwundert daher nicht, dass ein weiterer Stilist der Langsamkeit und des Innehaltens, nämlich Peter Handke, sich engagiert für das Werk von HERMANN LENZ eingesetzt hat. ${ }^{7} 1991$ gab er zusammen mit Peter Hamm und Michael Krüger eine Gedichtsammlung von LENZ bei Klaus G. Renner heraus.

2003 fragt sich Michael Krüger anlässlich der von ihm besorgten Lyrik-Anthologie in der Bibliothek Suhrkamp:

Gibt es gute (oder schlechte) Gründe dafür, daß dieser eigenwillige, weltlichfromme Dichter durch alle Siebe gefallen ist, die von den nicht besonders sorgfältigen Spezialisten des ,Was bleibt?' benutzt werden, um der Literaturgeschichte ihre philologische Physiognomie aufzuprägen? War man vielleicht der Ansicht, daß nach der freundlichen Aufforderung von Peter Handke (die gottlob wie ein Befehl wirkte), das erzählerische Werk von HERMANN LENZ zu lesen, genug getan worden sei für einen Autor, der, wenn es noch um einen ordentlichen, repräsentativen Sitz im Parnaß ging, doch eher eine Wiese bevorzugte, am Waldrand, mit einem freien Blick in die Ebene? Waren die Bescheidenheitsgesten die-

6 Ob sich dieser Zug, diese Neigung zum Detail, auch in der Lyrik wiederfindet, wäre an anderer Stelle zu diskutieren.

7 Obgleich selbst ein Starautor, teilt Peter Handke mit HERMANN LeNZ das Schicksal ebenfalls der Verfasser eines recht unbekannten lyrischen Werks zu sein. Auch dagegen versuchte der Suhrkamp-Verlag Abhilfe zu schaffen (vgl. HANDKE 2007). Mit LENZ teilt er - in der Lyrik wie auch in der eigenen Kurzprosa - die Vorliebe für das Festhalten von Impressionen, die durch Nuancen und Details ausgelöst und in eine höhere Bedeutung für den lyrischen Sprecher übersetzt werden. Vgl. unter anderem die Texte Gelegenheitsgedicht und Vollmond (HANDKE 2007:131f.). 
ses lauteren (und später natürlich auch selbstbewußten, was sich nicht ausschließen muß) Dichters so überdeutlich, daß man ihn der etwas lauteren Konkurrenz nicht aussetzen wollte? Fragen über Fragen, die von den gewitzten Literaturhistorikern und Anthologisten beantwortet werden sollten - oder auch nicht, wie Hermann Lenz gesagt hätte, der in dieser Hinsicht ohne Illusionen war: ,Vielleicht gelingt's und du überlebst./ Nein, sie machen dich fertig. '(KRÜGER 2003:147)

Fragen über Fragen, in denen immer auch die Kanonproblematik mitschwingt, die sich mit der Repräsentativität der Texte für die jeweiligen Autoren:innen, Werkkomplexe, Epochen und den zeithistorischen Hintergrund bzw. ihrer Bedeutung für Gegenwart und Zukunft auseinandersetzt. Gleichzeitig sind es Fragen, die den Autor LENZ in seiner Produktivität relativ kalt lassen. Ein kaum zu unterschätzender Pessimismus, verbunden mit einem Gespür für Augenblickserfahrungen, vor allem im Angesicht der Natur, die jedoch fernab aller mystischen und metaphorischen Überzogenheit in einer beinahe sinnlichen und greifbaren Konkretheit vollzogen werden, scheinen derlei Fragen obsolet werden zu lassen. Gegenüber politisch-intellektueller Positionierung weicht das Bedürfnis nach Rückzug bei gleichzeitiger Erkenntnis literarischer Eitelkeiten, die oft einher gehen mit eben diesen im Literatur- und Kulturbetrieb ausgelebten Potenzen. Mit letzterem ist ein Thema von LENZENS Werk benannt.

\section{Der Literaturbetriebsroman: Literarisches Feld und Textumgebung als Narrative}

Der Literaturbetriebsroman hat sich in den letzten Jahren, ähnlich wie der Campus- oder Professorenroman, als ein eigenständiges literarisches Genre durchgesetzt. Die marktinternen Regularien, welche den kontextuellen Hintergrund jeglicher literarischer Produktivität mit konfigurieren, haben seit den lakonischen Betrachtungen des Verlagswesens, der Genese von Popularität und der Bildung literarischer Zirkel wie der Gruppe 47 durch die Eugen Rapp-Romane von HERMANN LENZ ihre erzählerische Aneignung erfahren und eine Narrativierung des literarischen Feldes vollzogen.

Diese Transformation der Kontexte in den Text hatte vor allem in den letzten Jahren bei Autoren wie Walter Kempowski, Wolf Haas, Thomas Glavinic, Bodo Kirchhoff und Marlene Streeruwitz eine emphatische Selbstbehauptung von Auktorialität und Autofiktion auf der Erzählebene zur Folge, indem die komplexen und feldspezifischen (De- und) Konstituierungsmechanismen von Autorschaft durch den Literaturbetrieb nicht nur zum literarischen Stoff, sondern auch in narrative Verfahren umgesetzt wurden. Mag Hegel angesichts der Ökonomisie- 
rung und Marktregulation des künstlerischen Feldes in seinen Vorlesungen über die Ästhetik bereits im frühen 19. Jahrhundert vom Tod der Kunst gesprochen haben, Künstler und Künstlerinnen sind bis heute zum Glück noch nicht gestorben bzw. verschwunden. Paratexte und Interviews zeigen zum Beispiel seine Versuche sich auch unter poststrukturalistischen, postsubjektiven und postfaktischen Vorzeichen auktorial durchzusetzen und auf dem Markt, aber doch auch ganz klar für sich, Aufmerksamkeit über die Auto-Präsentation in der Öffentlichkeit als Anhäufung von symbolischem Kapital zu produzieren ${ }^{8}$ bzw. die eben genannten Prozesse als Narrative auszugestalten.

Dass sich dabei auch immer eine Reflexionsebene gegenüber dem literarischen Feld ergibt, ja letzteres und damit den Literaturbetrieb zum Narrativ werden lässt, wird anhand von HERMANN LENZ genauer herausgearbeitet. Durch die Auseinandersetzung mit dem Literaturbetrieb ist es möglich, die Inszenierung und Selbstbehauptung von Autorschaft und Autorschaftsbewusstsein zu reanimieren. Nach Manfred Engel rekonstruiert daraufhin der Literaturwissenschaftler diese Kontexte „durch die Interpretation von Texten und er perspektiviert sie durch seine interpretierende Rekonstruktion der Interpretation oder auch Modifikation, die der Autor des fokussierten Textes ihnen gegeben hat" (ENGEL 2018:74). ${ }^{9}$ Das gilt nicht nur für die intertextuellen Geflechte, in welche die Textproduktion inkludiert ist, sondern auch für die Produktionsbedingungen, unter denen der Text im Literaturbetrieb entsteht (oder vergeht), was ein Ineinanderweben von Fiktionalität- und Faktualitätserzählungen impliziert.

Sogenannte (Literatur-)Betriebsromane liefern zumeist in Form von Schlüsselromanen immer wieder interne Einblicke in die Kommunikationsverhältnisse innerhalb des Feldes, ja sie arbeiten auch mit den feldinternen Verfahren, passen

8 Auf vergnügliche Weise beschreibt beispielsweise Thomas Glavinic in seinem auktofiktionalen Roman Das bin doch ich (2006), wie es dem Erfolgsschriftsteller Daniel Kehlmann - im Gegensatz zum Ich-Erzähler - stets gelingt, die symbolische Kapitalakkumulation durch immer wieder neue Erfolge im Bereich der Arriviertheit zu legitimieren oder auch zu bestätigen. Das sogenannte Matthäus-Prinzip wird in diesem fiktiven Vergleich erhärtet.

9 Engel attestiert bei zahlreichen Autoren der Gegenwartsliteratur ein bewusstes Schreiben innerhalb dieser Kontexte, zu denen eben auch die feldspezifischen Produktions- und Rezeptionsbedingungen von Literatur zu zählen sind. Man versucht nicht mehr diesen, ästhetizistisch`zu entfliehen, sondern macht sie selbst zum Gegenstand literarischer Gestaltung bzw. man schreibt bewusst unter und mit diesen Diskursregimen. So werden Kontexte zu Texten und das literarische Feld birgt in sich ein ganzes Konglomerat an Erzählungen und Erzählweisen. 
ihre Erzählweise den strukturellen Determinanten des Literaturbetriebs an und transferieren diesen dadurch in einen narrativ gestaltbaren Diskurs der Mittelbarkeit. So ist HERMANN LENZ mit seinen Eugen Rapp-Romanen (als Autor jenseits des politischen Aktivismus und der intellektuellen Positionierung eines Grass oder Böll) ein Chronist zeittypischer Feldstrukturen. Die Gruppe 47 lädt ihn auch im Jahr 1951 ein, aber man findet nicht so recht zusammen. ${ }^{10}$ Der Roman Ein Fremdling von 1983 lässt eine mögliche Symbiose bereits vom Ansatz her scheitern, wenn auch recht vergnüglich:

Der schöne Mann riß die Augen auf und fügte nach einer Weile hinzu, der Verlag werde eine Tagung der Gruppe 47 in der Laufenmühle finanzieren. - ,Schreiben Sie an Richter, Sie wollen teilnehmen. Dem habe ich ihr Buch geschickt.' Da wurde es ihm dann mulmig zumute, weil er dachte, die Kollegen von der Gruppe seien alle so gewieft und so gerissen. Und warum dir dieser Betrieb zuwider ist, das weißt du auch nicht ... Schließlich hatten die schon einen namens Rapp, einen Ostpreußen, der Siegfried mit Vornamen hieß, und konnten deshalb nicht noch einen Eugen brauchen. Feine Kerle aber seid ihr beide, du und der Siegfried. (LENZ 1983:77)

Mit dieser Anekdote konfrontiert LENZ seine Leser immer wieder. Auch in anderen Romanen der Chronik wird sein alter Ego Eugen Rapp des Öfteren mit Siegfried Rapp/LENZ durch die Rezipientengemeinde verwechselt und erntet sogar Lob für wunderschöne Ostpreußenbücher und kritische Romane über den pflichtaffinen deutschen Polizeibeamten in der NS-Zeit. Die Regeln des Feldes und der aus ihnen resultierende Zwang nach Öffentlichkeit und Popularität zwecks Wahrnehmung von Autorschaft und die sich daraus ergebenden Erzählungen, mit einer deutlichen Affinität zur Mythisierung, funktionieren so gut (und automatisch), dass Rapp sogar für Texte einstehen könnte, die er niemals geschrieben hat. Der Name ist - freilich erst nach der Arrivierung - einfach alles und Dabeisein heißt für den Künstler nicht nur in soziologischer, sondern tatsächlich auch in ontologischer Hinsicht: Sein! (selbst wenn es Fake ist).

Hinzu kommt noch im Laufe des Romans: Der Schriftstellerverband (gemeint ist in den Romanen eventuell der P.E.N.-Club) schließt ihn später von seinem

10 Diese Phase findet vor allem ihre Aufbereitung im fünften Teil der autobiographischen Romanfolge um den Schriftsteller Eugen Rapp (vgl. LenZ 1983). Eine späte Periode literarischer Arrivierung im Kulturbetrieb und Verlagswesen dokumentiert, kaum mehr verschlüsselt, die finale Episode (vgl. LenZ 1997). Der Titel gebende Paratext Freunde ist ganz und gar unironisch gemeint und bezieht sich auf ein Panorama von literarischen Zeitgenossen wie Peter Handke und Peter Hamm, welche der Vermittlung LENZENS im Suhrkamp Verlag sehr hilfreich waren und mit denen er sich auch als Künstler verbunden fühlt. 
Sekretariatsposten (aus Altersgründen, aber auch wegen Divergenzen im Bereich der politischen Verantwortung von Schriftstellern) aus: Auch das Ego realiter LENZ teilt nicht die Begeisterung vieler Kollegen für die Regierung Brandt. Später wird er dann von Peter Handke, der ab den 1980er Jahren und vor allem in seinen tagebuchartigen Aufzeichnungen wie Phantasien der Wiederholung immer mehr die leisen Töne und die kondensierte Beobachtung und Erfassung von Seinsmomenten an die Stelle politisch orientierter Invektiven oder auch popkultureller Inszenierungen und Publikumsbeschimpfungen setzt, an Siegfried Unseld und damit an Suhrkamp vermittelt. Peter Handke macht 1973 in der Weihnachtsausgabe der Süddeutschen Zeitung mit seiner Einladung, Hermann Lenz zu lesen (HANDKE 1973) den Literaturbetrieb auf LENZ aufmerksam: einen „poetischen Geschichtsunterricht" meinte er zu entdecken, ,,voller Anmut, voller Würde.“ Er empfinde beim Lesen - „Glück“! Vielleicht weil er ebenfalls die Parameter der literarischen Feldes mit Skepsis betrachtet und damit zugleich eine intellektuelle Position und Erzählhaltung in absentia verteidigt, für die es in der europäischen Literatur der zweiten Hälfte des 20. Jahrhunderts auch Analogien zu finden gibt.

\section{Exkurs: Henry de Montherlant als Gegner der literarischen Öffentlichkeit?}

Es ist für das 20. Jahrhundert unter anderem der elitäre französische Schriftsteller und Romancier Henry de Montherlant, der über die Paradoxie von dichterischer Autonomie und Öffentlichkeit in seinem Tagebuch schreibt:

Der Umstand, daß das Kunstwerk zum Publikum gehen muß, ist die Kehrseite und die große Strafe für das erhabene Wunder der Kunst. Die Verbreitung des Werks verdirbt den Charakter des Künstlers und bringt ihn um die Zeit, die er seinem Werk und seinem Privatleben widmen sollte. (DE MONTHERLANT 1969:87) ${ }^{11}$

Zumindest wird hier deutlich, dass zum Beispiel auktoriale Paratexte ${ }^{12}$ und Interviews in diese paradoxale Ausgangslage kommunikativ, narrativ und performativ involviert sind, ganz gleich ob sie nun peritextuell oder epitextuell ausgerichtet

11 Dieser marktkritische Aphorismus wiederholt implizit die ebenfalls an Publikum und Auftragskunst gebundene Formel vom ,Tod der Kunst' durch Hegel. Zugleich rettet sich der Künstler, indem er innerhalb dieser ökonomisch-faktischen Parameter spricht und diese für sich kreativ zu nutzen weiß.

12 Die Forschungsliteratur ist stark angewachsen (vgl. GERSTENBRÄUN-KRUG / REINHARD 2018; Voss 2019). 
sind. Außerdem greifen Autorinnen und Autoren im Interview selbst auf inszenatorische Praktiken des Fingierens zurück und erschaffen damit sowohl verbal als auch gestisch und mimisch eine ganze Erzählung von sich selbst - und das mitten in einer scheinbar faktischen Erzähl- bzw. Gesprächssituation. Da die auktoriale Motivation besonders stark fokussiert wird, möchte ich noch folgenden Hinweis geben: Unterschlagen werden im Beitrag weitgehend die außerhalb der auktorialen Produktion stehenden Paratexte, die bei Genette unter die Begriffe der Metatexte oder auch der Allographen fallen, die aber nach Steffen Martus gerade mit Blick auf die Situation des Autors als Werkpolitiker Berücksichtigung finden sollten, denn

das Paratextzeremoniell aus Widmungen, Vorreden und Texten anderer Autoren deutet darauf hin, daß das Werk kein selbstbestimmtes Ineinander von Teil und Ganzen sein soll; es bildet keine wie auch immer bestimmte Totalität, sondern präsentiert sich als Schnittstelle von überindividuellen literarischen, politischen und sozialen Fertigkeiten, von allgemein verfügbaren Traditionen, Normen und Interessen. (MARTUS 2007:26)

Sie werden jedoch innerhalb des Literaturbetriebsromans oder des Feldromans, wie ich ihn vorschlagsweise nennen möchte, miteinander fusioniert. Zugleich wird auch aufgrund der Eingebundenheit all dieser Begleitmaterialien in die narrative und diaristische Kommunikation und in die verschiedenen Instanzen und Institutionen des literarischen Feldes, mit Thomas Wegmann gesprochen, auch etwas anderes deutlich:

Ob etwas als Kunst angesehen wird, ob ein gerade im Werden begriffener Text als ein literarischer Text gelesen wird, darüber entscheidet nicht der Künstler, sondern ein komplexer Kommunikationsprozess. (WEGMANN 2016:23f.)

HERMANN LENZ' alter Ego Eugen Rapp ist sich in den neun Romanen seines dichterischen Werdegangs und der damit verbundenen chronologischen Aufbereitung des Literatur- und Kulturbetriebs bis in die späten 1980er Jahre dieser Tatsache schmerzlich bewusst. Bei zahlreichen Autoren und Texten der Literatur ab 1945 fällt dieses Verhältnis beinahe dialektisch aus. Die Geburt der Kunst erfolgt zwar vermehrt aus dem Geiste der Ökonomie und der Praktiken des literarischen Feldes und setzt sich damit als eine Amalgamierung aus unterschiedlichen Diskursen zusammen. Zugleich hat der Autor als Inhaber einer poetologischen oder intellektuellen Position aber auch Anteil an der diskursiven Gestaltung und wird Segment von ihnen - und das auf eine recht reflektierte Weise, wie es unter anderem das Fallbeispiel HERMANN LENZ dokumentiert. Anders ausgedrückt: Dieser Einbindung in derartige Prozessualitäten und Diskursivitäten verdeutlichen sich die Autoren immer wieder. Und trotz aller teilweise auch hier verwendeten diskursanalytischen und feldtheoretischen Ter- 
minologie, lassen sich unterschiedliche Reaktionen des Autors und Erzählers LENZ auch als Versuche der auktorialen und subjektkonstituierenden Selbstbehauptung von Text und Künstler unter den medialen Gegebenheiten und innerhalb des Narrativs nicht leugnen, selbst (oder gerade) wenn diese die Unterlaufung feldinterner Regularien als Erzählstoff zum Gegenstand haben. Aus der zunehmenden Bewusstwerdung Teil eines komplexen Kommunikationsprozesses zu sein, erwachsen neue Modi der textuellen Gestaltung und der bewusst vollzogenen Einflussnahme oder auch Verweigerung innerhalb des literarischen Feldes. Man agiert immer unter und mit diesen Bedingungen auktorialer Möglichkeiten. ${ }^{13}$

So ist es wieder Henry de Montherlant, der im Juni 1962 Stellung zu einem filmischen Paratextprojekt nimmt, womit ein Regisseur von der bekannten französischen Filmfirma Gaumont einen Einblick in Montherlants Position im literarischen Feld und dessen eigene Selbstverortung präsentieren möchte. Ein solcher „Film erfaßt den Schriftsteller in seinem häuslichen Milieu, in seinem gewohnten Lebensumkreis, bei seinen Haupttätigkeiten usw." (DE MONTHERLANT 1968:126). Er bildet also einen buchfernen Epitext zum Gesamtwerk des Schriftstellers. Der elitäre Autor Montherlant betrachtet derlei Medialisierungen seiner Autorschaft jedoch skeptisch und kommt dabei auf Aspekte zu sprechen, die auch schon bei den Autorschaftsinszenierungen im literarischen Feld eine große Rolle spielen:

Es gibt sicher Schriftsteller, die das Talent haben, vor der Kamera natürlich zu bleiben. Ich habe, glaube ich, nicht dieses Talent; verfolgt von der Furcht, ich sähe so aus, als posierte ich, würde ich bestimmt zu guter Letzt posieren oder aber mich linkisch benehmen. Und außerdem, der grassierende Exhibitionismus: M. vor der Totenmaske Pascals. M., wie er mit zarter Hand seinen Plutarch (auf Papyrus) seiner zehntausendbändigen Bibliothek entnimmt. M. sitzend, im Akademiefrack, den Akademiedegen oder den des Matadors zwischen den Zähnen, wie er auf den Knien seine vierzehn Enkelkinder hält. Wer von Kameraleuten und Fotografen umringt ist, der ist von Teufeln umringt. (DE MONTHERLANT 1968:127)

Dem Tagebuch-Ich wird an dieser Stelle klar, dass die Mitwirkung an einer solche Inszenierung mit den eigentlichen poetologischen Anliegen divergiert und dass die auktoriale Popularität von anderen Kategorien abhängig ist. Durch die Verbindung der Journaille mit dem Attribut des Teufels erhält die gesamte Veranstaltung das Malediktum des Sakrilegs.

Obwohl das Autorenporträt in dieser Form nicht zustande kam, ist der Eindruck gegeben, Montherlant entwerfe - wenn auch widerwillig - geradezu ein

Insofern könnte man auch von der permanenten Anwesenheit eines Apriori sprechen. 
Drehbuch auktorialer Performanzen und Erzählungen, um seine Position innerhalb des Feldes zu bestimmen. Obgleich durch Gaumont nicht mehr visuell umgesetzt, ist es textuell im Tagebuch vorhanden und trotz seiner Verweigerungshaltung (oder gerade wegen ihr) radikal inszenatorisch. Auf jeden Fall verweist der Vorgang des Posierens auf die im Distinktionsbetrieb des literarischen Feldes so relevante Form des Rollenspiels, über welches Auktorialität erst sichtbar und erzählbar wird. Und Montherlant beschreibt mit dem filmischen Autorenporträt eine ganz typische feldspezifische Inszenierung, die auch in den Eugen Rapp-Romanen von HERMANN LENZ immer wieder kritisch beleuchtet auftauchen wird. Nichts verabscheut Rapp mehr als filmische Autorenporträts, deren Produzenten in unglaublicher Geschäftigkeit in seinen Lebensbereich einbrechen und ihm ein bestimmtes Bild vom Schriftsteller für die Öffentlichkeit aufzwingen. Aber: Man kann nicht nicht erzählen, um einmal den Linguisten und Philosophen Paul Watzlawick instrumentell abzuwandeln.

\section{Konstellationen, Konversationen und Machtfelder als Textumge- bungen?}

Ganz gleich ob von Kollegen des Autors oder von ihm betrieben; unerheblich ob es sich dabei um textferne Gespräche und Gerüchte, um Rezensionen, Interviews oder um Vorabdrucke jenseits der Bücher handelt, sie beeinflussen als Begleitmaterial die Wirkung von letzteren und ihrer Verfasser im literarischen Feld, was auch Pierre Bourdieu in seiner Feldanalyse schon für das ausgehende 19. Jahrhundert bestätigt:

Die der Zugehörigkeit zum Macht-Feld inhärenten Zwänge vollziehen sich auch innerhalb des literarischen Feldes zugunsten der Austauschbeziehungen zwischen den Mächtigen, mehrheitlich Aufsteigern auf der Suche nach Legitimität, und den konformistischen oder arriviertesten Schriftstellern, und dies vor allem über das subtil hierarchisch abgestufte Universum der Salons. (BOURDIEU 2001:81)

Solche Verfahren strukturieren Hierarchisierungen innerhalb des Machtfeldes und der ihm integrierten Möglichkeiten zur Arrivierung. ${ }^{14}$ Sie steuern die Re-

14 Ebenso präzise benennt Bourdieu die sich daraus ergebenden Konsequenzen: „Nicht selten ergeben sich auch über solche zwiespältigen Personen in prekären Lagen [...] sanfte Formen der Beeinflussung, die die völlige Abspaltung der Inhaber kultureller Macht verhindern oder entmutigen und diese Kulturträger in unüberschaubare Beziehungen verstricken, beruhend auf der aus dem Kompromiß und der Kompromittierung erwachsenden Dankbarkeit und dem Schuldgefühl einer fürsprechenden 
zeptionshaltungen und geben einen Eindruck von den Qualitäten und dem Selbstverständnis der Autoren, belanglos ob diese nun selbst epi- und peritextuell tätig sind oder andere, das literarische Feld und den Buchmarkt kontrollierende, Instanzen wie Verlage, Kritiker oder in der Öffentlichkeit angekommene Schriftstellerkollegen mitwirken.

Mit Bourdieu wird das für die Literaturwissenschaft plausibel,

wenn sie die Logik der Arbeit am Schreiben, verstanden als unter den Strukturzwängen des Feldes und der von ihm gebotenen Möglichkeiten durchgeführtes Erfinden, zu rekonstruieren unternähme. [...] Man verstünde das Zögern, das Verwerfen, die Rückkehr zu früheren Fassungen besser, wenn man realisierte, daß das Schreiben, diese gefährliche Schiffahrt in einem Ozean drohender Gefahren, in seiner negativen Dimension auch durch eine vorweggenommene Kenntnis der wahrscheinlichen, dem Feld als Möglichkeit innewohnenden Rezeption geleitet wird. (BOURDIEU 2001:315f.)

Diese wird zum Apriori der Schreibsituation, die jene Vorbedingung als Erzählung einfängt.

Die durch LENZENS alter Ego Eugen Rapp vollzogene Figuralisierung und Narrativierung von Autorschaft und Feld bewirkt eine satirisch-sarkastische Durchleuchtung eben dieser von Bourdieu genannten Strukturzwänge der Funktionsweisen und Mechanismen des literarischen Feldes über mehrere Jahrzehnte hinweg. Sie ergibt aufgrund der Zyklizität der Romanabfolge eine chronistisch-narrative Bestandaufnahme des Literaturbetriebs der Bundesrepublik. Ziel ist dabei die Illumination der Konstituierung der sozialen Felder als „relationale Machtkonfigurationen [...], in denen sich je eigene historisch gewordene soziale Logiken und Regelsysteme ausgebildet haben" (FüsSEL 2005:189), wie es Marian Füssel unter Rekurs auf Bourdieus Theorie für die Konstellationsmechanismen auch in intellektuellen und akademischen Feldern herausgestellt hat.

Die von LENZ in verschlüsselter Form in sein Romanwerk aufgenommenen Repräsentanten des zeitgenössischen Literatur- und Kulturbetriebs dienen der Allego-

Macht gegenüber, die als letzte Zuflucht oder zumindest doch als eine Ausnahmeinsel wahrgenommen wird“ (BOURDIEU 2001:89). Einmal abgesehen davon, dass sich ähnliches auch im akademischen Feld vollzieht, wäre zu klären, inwieweit auch auktoriale epitextuelle Textangebote eine solche Wirkung entfalten können und welchen Beitrag sie innerhalb dieser Mechanismen zur Konstituierung von Autorschaft leisten können. Vgl. zur Feldtheorie auch den Reader von PiMTo / SchUltheIs (1997). 
risierung und damit der Verdeutlichung (im Sinne von Komplexitätsreduktion) ${ }^{15}$ all dieser Mechanismen, Funktionen und Funktionsträger, an denen er auch selbst partizipiert. Trotz aller Beharrung auf autonomer Autorschaft und damit inkludierter Verweigerungsstrategien durch Autoren wie HERMANN LENZ (und seinen Entdecker und Förderer Peter Handke) ist mit Füssels Bourdieu-Rekurs zu beachten:

Die Akteure eines Feldes sind in ihrem Denken und Handeln durch die ,Struktur der objektiven Beziehungen', wie etwa den unterschiedlichen Zugang zu Ressourcen, zu den anderen Akteuren des Feldes bestimmt. Es geht demnach nicht um die Position innerhalb des sozialen Raumes als ganzem, sondern um die spezifische Position innerhalb des Feldes. [...] Vor dem Hintergrund des differenztheoretischen Axioms, daß ein Element nur innerhalb der relationalen Struktur, zu deren Konstitution es selber beiträgt, seine Bedeutung erhält, müssen auch die Texte und Akteure einer Konstellation nicht aus sich heraus bedeutungstragend begriffen werden, sondern im Kontext ihrer feldspezifischen Stellung, also nur in Abgrenzung zu anderen Positionen. (FüSSEL 2005:191)

Bedeutung entsteht also innerhalb der Interaktion der Feld-Akteure bzw. auch Feld-Antagonisten und ihrer spezifischen Rezeptionshaltungen, welche die Zugehörigkeiten innerhalb des Feldes mit bestimmen.

\section{HeRmann LenZ und die Regularien des Feldes: Die Gruppe 47, der elektrische Stuhl und Eugen Rapps Lesung in Ein Fremdling (1983) - eine erzählte Feldforschung?}

Wie die genannten Parameter bei LENZ nun in textualisierter Form funktionieren und welche Bedeutung sie innerhalb der Inszenierung von Autorschaft innehaben, wird in den satirischen und selbstkommentierenden Bemerkungen des Romanciers deutlich. Trotz aller konstellativer Mechanismen des literarischen Feldes, zu welchen auch die eigentlichen Romanpublikationen einen Beitrag leisten, sind letztere nach Manfred Beetz doch auch Erzählweisen und damit „Kommunikationsmittel, deren spezifische Funktionen aus der subjektiven Perspektive der Akteure und objektivierbaren Feld-Strukturen zu ermitteln sind" (BEETZ 2003:35). Gerade bei einem in Narrativen der sowohl stilistisch als auch inhaltlich-stofflich vollzogenen Verweigerung arbeitenden und be-

15 Womit ein seit Georg Lukács Theorie des Romans (1920) bestehendes Anliegen des Romans implizit durch Hermann LenZ wieder aufgegriffen wird. Die Ironie besteht freilich darin, dass die Aufdeckung der Komplexität durch die großen Romane erstere nicht beseitigt sondern nur bestätigt. 
wusst auf Distinktion und Habitualisierung setzenden Autor wie LENZ ist die von Beetz gestellte Forderung nicht unerheblich. Das Verhältnis von distinguierter Separation und kontextueller Einbindung ist nicht leicht zu konfigurieren, denn auch die literarische Praxis ist, mit Bourdieu gesprochen, ,weder vollständig autonom noch einseitig durch externe gesellschaftliche Faktoren, wie politische oder ökonomische Imperative bestimmt, sie ist vielmehr in sich selbst eine genuin soziale Veranstaltung“ (BOURDIEU 2001:20). Und LENZ bewegt auch diese bzw. wird von dieser bewegt - gerade in seiner beobachtenden, decouvrierenden, chronologisierenden, kommentierenden und teilweise oppositionellen Haltung (und auktorialen Inszenierung) gegenüber den feldtypischen Regularien, wie es eine erste Feld- und Selbstanalyse aus dem Roman Der Fremdling offenbart:

Eigentlich nicht schlecht, nur daß du leider alles umsonst machen mußt ... Und es grauste ihm vor dem Adressenschreiben; auch mußte er mit dem Kleinen und Bebrillten mindestens zweimal in der Woche telefonieren. All diese Kollegen waren aus Berlin oder aus Norddeutschland, der ,Sowjetzone', wie man jetzt sagte. Daß die Eugen akzeptierten (dich, einen ungeschickten, einen immer wieder geistesabwesenden Schwaben) und nahezu froh an ihm waren, dies verwunderte ihn. Von heut auf morgen konnte es sich freilich ändern. Oder würden die einen wie dich nicht im Handumdrehen finden? Ein ,echter' Schriftsteller bist du deshalb nicht, weil du nicht aufmuckst (au net schlecht). Über den etwas Abfälliges und über jenen Lobendes ins Gespräch zu mischen, um ein Beziehungsgeflecht wie ein Netz auszuspannen, in dem sich seine Feinde fingen, dafür war Eugen nicht geschaffen; er kannte auch von den Schriftstellern so gut wie keinen. Zu wenig vif und aufgeweckt bist du dazu, vielleicht auch zu empfindlich, dachte er. Und du weißt nicht, wie man ein solches Intrigen-Gespinst flicht. Freilich, mühsam hast du's schon, weil du den Bürokram machen mußt, dich aber lieber auf dich selbst besinnen würdest. Doch letzten Endes kam es nicht so genau darauf an (wie auf den falschen Konjunktiv). [...] Immer wieder vergißt du, daß du Schriftsteller bist ... sagte Eugen zu sich selber, machte tagsüber Bürodienst als ein Sekretär und kletterte abends in seine Dachstube hinaus, um zu kritzeln. Es wollte aber nichts Gescheites daraus werden. (LENZ 1983:70, 76)

Rapp erläutert an dieser Stelle feldinterne Praktiken der Anhäufung symbolischen Kapitals ${ }^{16}$ und auch der Machtverschiebung, die nicht unbedingt mit einer

16 Bourdieu hat ähnliche Mechanismen und Strukturen innerhalb des akademischen Feldes benannt: „Universitäres Kapital erhält oder behält, wer Positionen innehat, mit denen sich andere Positionen und deren Inhaber beherrschen lassen. Dazu zählen die Institutionen, denen die Kontrollen des Zugangs zur Körperschaft anvertraut sind““ (BouRdiEu 2018:149). Ergo: Stellen und Preise bekommt diejenige/derjenige, die/der bereits über Preise und Stellen verfügt. 
poetologischen oder intellektuellen Position korrelieren müssen. Oft gilt auch das als Position, was anerkannt und in der kulturellen Öffentlichkeit arriviert oder zumindest zur Kenntnis genommen ist - und sei es als Opposition. Jedoch ohne das symbolische Kapital der Popularität ist derlei kaum möglich. Rapps Unterlaufung dieser feldspezifischen Reglements der Macht ergibt sich einerseits aus der simplen Tatsache ihrer Unbeherrschbarkeit durch den Schwaben. Andererseits aber bewirkt genau dieser feldpolitische Mangel die Kultivierung einer alternativen Ausgestaltung von Distinktion, die sich eben daraus speist, kaum wahrgenommen zu werden, jedoch selbst so einiges wahrnehmen zu können.

Sie wird im Roman Ein Fremdling auch Gegenstand des Erlebnisberichts über eine Lesung vor der Gruppe 47 auf dem sogenannten elektrischen Stuhl, die zu den luzidesten Einblicken in den Literaturbetrieb, oder man müsste besser sagen, in den literarischen Markt zu zählen sind, die von HERMANN LENZ erstellt wurden. Sie benennen Praktiken und Praktiker der Distinktion, die sich über Verfahren der sozialen Differenzierung und damit über habituelle Arriviertheit zu einer Art Zitierkartell zusammenschließen, welches sich - den von Bourdieu beobachteten literarischen Salons und Avantgardezirkeln der Jahrhundertwende nicht unähnlich ${ }^{17}$ - durch eigene Parameter der Kommunikation zu akzentuieren weiß. Über Modi der Exklusion setzt es sich öffentlich, denn es geht um Visualität und Performativität, also um sichtbare Erzählungen, in Szene:

Dann also auf den ,elektrischen Stuhl‘. Er las drei Seiten [...] und hernach sagte einer, der vom Vorsteher nach seiner Meinung gefragt worden war, er habe ,rein akustisch" nichts verstanden. Ein zweiter sagte, das Gelesene sei nichts, er wundere sich nur, daß zuvor all dies so groß angekündigt worden sei [...]. Ein älterer Herr, der emigriert gewesen war, sagte, er halte es für eine große Arbeit von genialer Simplizität. Zwei Damen tuschelten schnippisch und lachten leise. Ein Pudel, der bei einem Mann mit Knotenstock lag, blaffte, und es hieß, das sei ein literarischer Hund; wenn etwas Schlechtes vorgelesen sei, dann belle der. [...] Und Eugen dachte wieder einmal: kurios. (LENZ 1983:80)

Letzteres ist eine Wertung der kaum durchschaubaren Verflechtungen und zugleich eine intertextuelle Anspielung auf das Sterbewort des alten Konsuls Buddenbrook, mit dem er sich in Thomas Manns Familienroman von 1901 zur Wand dreht und aus der Welt zurückzieht.

Damit markiert „kurios“ auch die Einnahme von Distanz. Auf die Frage, wann ein gewisser Brumme zur Gruppe 47 gestoßen sei, kontert er mit dem Gedanken: „Da stieß man also zur Gruppe wie seinerzeit zur Partei“ (LENZ 1983:84) und

17 Falls man die Gruppe 47 einem Salon gleichsetzen möchte. Man kam nur über Einladungen H.W. Richters hinein. 
benennt funktionale Äquivalenzen innerhalb der Strukturen des politischen und des literarischen Feldes. Zugehörigkeit wird von mehr gesteuert als von kreativen Potentialen. Dass selbst die scheinbar einfache und minimalistische Wiedergabe der kommunikativen und sozialen Verhältnisse der Gruppe 47 einen sukzessiven erzählerischen Ablauf ergeben können, hat Helmut Heißenbüttel mit seiner Gruppenkritik im Textbuch 5 so stark pointiert, dass sich diese kurze Prosa beinahe wie ein Kondensat der Feld- und Schlüsselromane von HERMANN LENZ lesen lässt (vgl. HeißENBÜTTEL 1980:227-229). Die sich daraus ergebenden Folgen für den Ex-Patriierten LENZ bzw. Rapp und dessen eigenes Selbstverständnis als Schriftsteller finden ebenso ihren Niederschlag im Gedicht Rückblick aus der mittleren Schaffensperiode um 1960:

Kein Haus gebaut

Keinen Sohn gezeugt,

Nur Bücher geschrieben.

Genügt es?

Nein, es genügt nicht.

Auch das mit dem Besitz

Ist bei dir so eine Sache,

Eine fragwürdige, wie sich versteht.

In Dachstuben hast Du gewohnt

Mit Möbeln von früher.

Die hast du lange gekannt.

Was die andern so ,Leben“ nennen,

War für dich mühsam.

Geschafft hast du es nie.

Wenn du nur durchkommst. (LENZ 2003:86)

\section{Auswertung: Erzählen im Literaturbetriebsroman/Feldroman durch HERMANN LENZ}

Aus einem Zusammenfall von klarer Feldschau, Transformation der feldinternen Mechanismen in Narrative und damit zugleich erkannter als auch schlussendlich behaupteter Position im Literaturbetrieb ergibt sich eine einfache, lyrisch gefasste Konsequenz für den Künstler im späten Gedicht Versteinerung, worin es in den letzten Versen heißt: „Ein großes Unglück, daß der Mensch intelligent ist, / Während der Farn nicht mehr will / als hier sein für kurze Zeit. // Vielleicht lebst du weiter im Stein“" (LENZ 2003:61). LENZ war noch hier bis 
1998 und der Verweis auf den Stein als niederste Existenzform in der hinduistischen Kette der Wiedergeburten, ist zugleich ein impliziter metaphorischer Hinweis auf die Inszenierung von utopischer Beständigkeit jenseits des Feldes und der Öffentlichkeit und synchron die konstitutive Voraussetzung für die Einnahme einer distanzierten Erzählperspektive, von der es aus (auktoriale) Narrative zu erkennen und zu gestalten gilt. Denn diese wechseln ja ständig und sind gebunden an ihre Praktiken der Distinktion. Und deren Betreiber:innen bleiben austauschbar, während der Stein einfach nur bleibt. So verwundert es nach Michael Krüger nicht, dass LENZ

in seinem autobiographischen Romanwerk, der Zeit ins Gesicht sehen mußte, um Distanz zu schaffen zwischen seinem Lebenslauf und dem Lauf der Geschichte. [...] Es gibt wohl keinen Dichter seiner Generation, der illusionsloser und härter, also unsentimentaler, den geschichtsphilosophischen oder theologischen oder auch nur alltäglich-lebenspraktischen Versprechungen widersprochen hätte. Nein, nicht einmal Widerspruch meldet er an. Widerspruch wäre bereits ein Einlassen auf die Gesetze und Regeln, bedeutet ein aktives Teilnehmen, ein Eingreifen, also das Gegenteil dessen, was er selbst für sich beansprucht. (KRÜGER 2003:149f.)

Das würde für LENZ die Aufgabe des Beobachtens und damit des Erzählens implizieren. Denn Beobachten und Durchschauen sind nicht nur die konstituierenden Voraussetzungen a priori für die Analysen literarischer, kultureller und auch intellektueller Felder. Sie sind das Erzählen selbst.

Freilich ergibt sich daraus die Frage, welche Position LENZ bezieht. Kann diese denn nun auch im Abseits liegen oder ,im inneren Bezirk“?: „Was du redest, hörst nur du selbst [...] Dir fällt ein, / Daß du allein bist und schreibst. / An wen? An dich selber [...] Leg die Feder beiseite/Und schau vor dich hin“"(LENZ 2003:82). Letzteres müsste jedoch synchron heißen, so paradox es angesichts der beiseitegelegten Feder anmuten mag: Und schreib vor dich hin - und erzähle! Als Eugen Rapp tat HERMANN LENZ genau das.

\section{Literatur}

BeEtz, MANFRed (2003): Konversationskultur und Gesprächsregie in den Monatsgesprächen. In: BEETz, MANFRED / JAUMANn, HERMANN (eds.): Thomasius im literarischen Feld. Neue Beiträge zur Erforschung seines Werkes im historischen Kontext. Tübingen, 35-60.

Bourdieu, PierRe (2001): Die Regeln der Kunst. Genese und Struktur des literarischen Feldes. Aus dem Französischen v. Bernd Schwibbs und Achim Nusser. Frankfurt a.M. Bourdieu, Pierre (1988/2018): Homo academicus. Aus dem Französischen von Bernd Schwibs. Berlin / Frankfurt a.M. 
BRIEGLEB, Klaus (1992): Weiterschreiben! Wege zu einer deutschen literarischen ,Postmoderne'. In: BriegleB, Klaus / WeIGel, SigRID (eds.): Hansers Sozialgeschichte der deutschen Literatur vom 16. Jahrhundert bis zur Gegenwart. Bd. 12. Gegenwartsliteratur seit 1968. München, 340-381.

Engel, MANFred (2018): Kontexte und Kontextrelevanzen in der Literaturwissenschaft. In: KulturPoetik 1:71-89.

Franzen, JoHANnes (2018): Indiskrete Fiktionen. Theorie und Praxis des Schlüsselromans 1960-2015. Göttingen.

FÜSSEL, MARIAN (2005): Intellektuelle Felder. Zu den Differenzen von Bourdieus Wissenssoziologie und der Konstellationsforschung. In: Mulsow, MARTIN / STAMM, MARCELO (eds.): Konstellationsforschung. Frankfurt a.M., 188-206.

GerstenbräUn-Krug, Martin / NADJA Reinhard (2018) (eds.): Paratextuelle Politik und Praxis. Interdependenzen von Werk und Autorschaft. Wien.

HANDKe, Peter (1973): Tage wie ausgeblasene Eier. Einladung, Hermann Lenz zu lesen. In: Süddeutsche Zeitung (22./23. Dezember 1973).

HandKe, Peter (2007): Leben ohne Poesie. Gedichte. Frankfurt a.M.

Heißenbüttel, Helmut (1980): Gruppenkritik. In: DerS.: Textbücher 1-6. Stuttgart, 227-229.

KreuZer, Ingrid / KreuZer, Helmut (eds.) (1981): Über Hermann Lenz. Dokumente seiner Rezeption (1947-1979) und autobiographische Texte. München.

KRÜGER, Michael (2003): Nachwort. In: LenZ, Hermann: Vielleicht lebst du weiter im Stein. Gedichte. Frankfurt a.M., 147-155.

LenZ, Hermann (1983): Ein Fremdling. Roman. Frankfurt a.M.

LENZ, HERMANN (1997): Freunde. Roman. Frankfurt a.M. / Leipzig.

LENZ, HERMANN (2003): Vielleicht lebst du weiter im Stein. Gedichte. Frankfurt a.M.

MARTUS, STEFFEN (2007): Werkpolitik. Zur Literaturgeschichte kritischer Kommunikation vom 17. bis ins 20. Jahrhundert mit Studien zu Klopstock, Tieck, Goethe und George. Berlin.

De Montherlant, Henry (1968): Geh, spiel mit diesem Staub. Tagebücher 1958-1964. Aus dem Französischen v. Karl August Horst. Köln / Berlin 1968.

DE MONTHERlant, Henry (1969): Tagebücher 1930-1940. Aus dem Französischen v. Karl August Horst. München.

Pimto, Louis / SchultheIs, FRAnz (eds.) (1997): Streifzüge durch das literarische Feld. Texte von Pierre Bourdieu, Christophe Charle, Moulaud Mammeri, Jean-Michel Péru, Michael Pollak, Anne-Marie Thiesse. Konstanz (Edition discours; Bd. 4).

Rösch, GerTRud MARIA (ed.) (2005): Codes, Geheimtext und Verschlüsselung. Geschichte und Gegenwart einer Kulturpraxis. Tübingen.

VOß, TORSTEN (2019): „,Drumherum geschrieben? “. Zur Funktion auktorialer Paratexte für die Inszenierung von Autorschaft um 1800. Hannover.

WAGNeR-EGelHAAf, MARTINA (ed.) (2013): Auto(r)fiktion. Literarische Verfahren der Selbstkonstruktion. Bielefeld. 
Wegmann, Thomas (2016): ,Es stimmt ja immer zugleich alles und nichts': Zur Theorie des Autors und zum Tod als Gegenstand in Interviews: Müller, Bernhard, Derrida. In: The German Review: Literature, Culture, Theory 91:7-24. 